PAPER

\title{
Incidence and diagnosis of anosognosia for hemiparesis revisited
}

\section{B Baier, H-O Karnath}

J Neurol Neurosurg Psychiatry 2005;76:358-361. doi: 10.1136/jnnp.2004.036731

See end of article for authors' affiliations .....................

Correspondence to: Prof Hans-Otto Karnath, Centre of Neurology, Hertie-Institute for Clinical Brain Research, University of Tübingen, HoppeSeyler-Str 3, D-72076 Tübingen, Germany; Karnath@uni-tuebingen.de

Received 14 January 2004 In revised form 10 June 2004

Accepted 10 June 2004

Background: In previous studies, the incidence of anosognosia for hemiparesis has varied between $17 \%$ and $58 \%$ in samples of brain damaged patients with hemiparesis.

Objective: To determine whether this wide variation might be explained by the different criteria used for diagnosing anosognosia.

Methods: 128 acute stroke patients with hemiparesis or hemiplegia were tested for anosognosia for hemiparesis using the anosognosia scale of Bisiach et al.

Results: $94 \%$ of the patients who were rated as having "mild anosognosia" - that is, they did not acknowledge their hemiparesis spontaneously following a general question about their complaintssuffered from, and mentioned, other neurological deficits such as dysarthria, ptosis, or headache. However, they immediately acknowledged their paresis when they were asked about the strength of their limbs. Their other deficits clearly had a greater impact. These patients had significantly milder paresis than those who denied their disorder even when asked directly about their limbs.

Conclusions: Patients who do not mention their paresis spontaneously but do so when questioned about it directly should not be diagnosed having "anosognosia." If this more conservative cut off criterion is applied to the data of the present as well as previous studies, a frequency of between $10 \%$ and $18 \%$ for anosognosia for hemiparesis is obtained in unselected samples of acute hemiparetic stroke patients. The incidence thus seems smaller than previously assumed.

S troke patients with anosognosia for hemiparesis typically deny their paresis or behave as if the disorder did not exist. They are convinced that their paretic or plegic limb functions normally. They may address their paresis, but then consider it as harmless and state that, for example, their leg "is tired". Anosognosia is not only found in hemiparesis; it may also be observed with cortical blindness, hemianopia, or deafness. ${ }^{12}$

Previous studies have shown wide variation in the incidence of anosognosia for hemiparesis. Bisiach et a ${ }^{3}$ reported that about 33\% of right brain damaged patients had medium or severe anosognosia for hemiparesis. Cutting ${ }^{4}$ found that $58 \%$ of right brain damaged patients had anosognosia for hemiparesis, while Maeshima et al reported only $24 \%$ with this sign. In a study by Starkstein et al, ${ }^{6} 31 \%$ of right and left sided acute stroke patients had anosognosia for hemiparesis. Other studies reported incidences ranging from $17 \%$ to $28 \%$ for the same disorder. ${ }^{7-9}$

We wondered whether this wide variation in the incidence might reflect varying criteria for diagnosing anosognosia for hemiparesis. All assessment scales applied in previous studies $^{3410}$ used a short interview to consider a patient anosognosic for hemiparesis. However, so far none of the scales has had general acceptance as the standard procedure. The questionnaires suggested by Bisiach $e t \mathrm{al}^{3}$ and by Cutting have been widely distributed and frequently used in clinical studies. ${ }^{6} 9^{11} 12$ They both start with a general question about the patient's complaints. The advantage of Bisiach's questionnaire $^{3}$ is that it allows classification of the degree of anosognosia into "mild", "medium", and "severe", while the scale of Cutting ${ }^{4}$ only uses a dichotomous classification (having anosognosia $v$ not having anosognosia).

According to Bisiach's scale, "mild anosognosia" for hemiparesis is diagnosed when patients do not acknowledge their paresis when posed a general question about their symptoms. In this study we ask whether such behaviour should indeed be regarded "pathological" or whether it is still within the normal range (especially when hemiparesis is not the only symptom involved after a stroke). Should patients who do not mention their paresis but complain spontaneously of other deficits be considered as having "anosognosia for hemiparesis"?

To investigate this question we asked how patients with "mild" anosognosia differ in their clinical variables from patients with "medium" or "severe" anosognosia. We further asked whether it is possible that patients with "mild" anosognosia do not acknowledge their paresis spontaneously because they have other deficits that may have a greater impact subjectively.

\section{METHODS}

We investigated patients with circumscribed right and left hemisphere lesions consecutively admitted from a well defined area belonging to the University of Tübingen and six related community hospitals over a period of six months. We included patients who had developed acute stroke within the previous 15 days, with left or right sided hemiparesis or hemiplegia. Lesions were documented by magnetic resonance imaging or computed tomography. Patients who were not alert, not cooperative, or had severe aphasia were excluded only if their communication abilities did not permit a simple interview for anosognosia (see below).

The study group comprised 128 patients with hemiparesis or hemiplegia, 72 with right brain damage and 56 with left brain damage (table 1). The patients gave informed consent for their participation in the study, which was done in accordance with the ethical standards laid down in the 1964 Declaration of Helsinki. All examinations were carried out immediately after admission-that is, in the acute phase of the stroke.

The degree of paresis of the upper and lower limbs was scored with the usual clinical ordinal scale, where 0 is no 
Table 1 Demographic and clinical data on all brain damaged patients with hemiparesis or hemiplegia

\begin{tabular}{|c|c|c|c|c|c|}
\hline Variable & & Denial grade 0 & Denial grade 1 & Denial grade 2 & Denial grade 3 \\
\hline Number & & 99 & 17 & 8 & 4 \\
\hline $\operatorname{Sex}(M ; F)$ & & $54 ; 45$ & $6 ; 11$ & $6 ; 2$ & $3 ; 1$ \\
\hline Age (years) & Median (range) & $69(20$ to 100$)$ & 70 (45 to 89 ) & $71(51$ to 84$)$ & $78(54$ to 91$)$ \\
\hline Aetiology & & 84 inf, 15 haem & 12 inf, 5 haem & 3 inf, 5 haem & 3 inf, 1 haem \\
\hline Time since lesion (days) & Median (range) & $4(0$ to 14$)$ & $4(0$ to 11$)$ & 3.5 (1 to 15$)$ & $3(0$ to 14$)$ \\
\hline \multicolumn{6}{|l|}{ Side of lesion } \\
\hline Right & $\%$ present & 56 & 41 & 75 & 100 \\
\hline Left & $\%$ present & 44 & 59 & 25 & 0 \\
\hline Paresis of contralesional side & $\%$ present & 100 & 100 & 100 & 100 \\
\hline Arm & Median (range) & $4(0$ to 4$)$ & $3(0$ to 4$)$ & $0(0$ to 4$)$ & $0(0$ to 1$)$ \\
\hline Leg & Median (range) & $4(0$ to 4$)$ & $3(0$ to 4$)$ & $0(0$ to 3$)$ & $0(0$ to 3$)$ \\
\hline \multirow[t]{2}{*}{ Visual field defects } & $\%$ present & 4 & 0 & 13 & 0 \\
\hline & $\%$ tnp & 3 & 6 & & \\
\hline \multirow[t]{2}{*}{ Neglect } & $\%$ present & 16 & 24 & 75 & 75 \\
\hline & $\%$ tnp & 13 & 12 & 13 & 25 \\
\hline \multicolumn{6}{|l|}{ Extinction } \\
\hline \multirow[t]{2}{*}{ Visual } & $\%$ present & 19 & 52 & 75 & 75 \\
\hline & $\%$ tnp & 5 & 6 & 13 & \\
\hline \multirow[t]{2}{*}{ Tactile } & $\%$ present & 22 & 41 & 75 & 75 \\
\hline & $\%$ tnp & 4 & 12 & 13 & \\
\hline \multirow[t]{2}{*}{ Auditory } & $\%$ present & 19 & 18 & 75 & 75 \\
\hline & $\%$ tnp & 6 & 12 & 13 & \\
\hline Aphasia & $\%$ present & 14 & 24 & 0 & 0 \\
\hline MMSE & Median (range) & 25 (9 to 30$)$ & 18.5 (6 to 29 ) & $13.5(10$ to 21$)$ & 15.5 (4 to 24$)$ \\
\hline
\end{tabular}

Denial grade 0 to 3 according to the anosognosia scale of Bisiach et al. ${ }^{3}$

$\mathrm{f}$, female; haem, haemorrhage; inf, infarct; $\mathrm{m}$, male; MMSE, mini-mental state examination ${ }^{13}$; tnp, testing not possible.

trace of movement and 5 is normal movement. Scoring was carried out separately for the proximal (upper arm, thigh) and distal (forearm, lower leg) parts of each limb. Table 1 presents the averaged scores per limb.

Anosognosia for hemiplegia was examined using a German translation $^{14}$ of the anosognosia scale suggested by Bisiach et $a l^{3}$ :

- grade 0 (no anosognosia): the disorder is spontaneously reported or mentioned by the patient following a general question about their complaints;

- grade 1: the disorder is reported only following a specific question about the strength of the patient's limbs;

- grade 2: the disorder is acknowledged only after demonstrations through routine techniques of neurological examination;

- grade 3: no acknowledgement of the disorder can be obtained.

Visual field defects were assessed by standard neurological examination. Spatial neglect was diagnosed when the patients showed characteristic clinical behaviour such as orienting towards the ipsilesional side when addressed from the front or the left, and/or ignoring contralesionally located people or objects. In addition, all patients had to show spatial neglect in the bells test. ${ }^{15}$ This test consists of seven columns each containing five targets (bells) and 40 distractors. Three of the seven columns are on the left side of the DIN A4 sheet ( = 15 targets), one is in the middle, and three are on the right ( $=15$ targets). The patient is asked to cross out all the bells. Omission of more than five bells within the three contralesional columns compared with the three ipsilesional columns is considered to indicate spatial neglect.

The patients were clinically tested for visual, auditory, and tactile extinction. In each mode, 10 unilateral stimuli on either side and 10 bilateral stimuli were presented in a pseudo-random order. Visual extinction was tested by the usual clinical confrontation technique. Movements of the examiner's left or right index finger were presented in the patient's left or right visual half field. Auditory and tactile stimulation tests were conducted with the patient's eyes closed. The auditory mode was tested by rustling a small piece of paper near the patient's left or right ear. Tactile extinction was investigated by applying fingertips lightly on the dorsal surface of the patient's left or right hand while the patient's arms lay relaxed. If the patient was not able to report this gentle unilateral tactile stimulation on the contralateral hand owing to left sided sensory loss, the examination was repeated using soft pinching applied to the left or right shoulder. Patients were classified as showing extinction in the respective mode when they reported $\geqslant 90 \%$ of the unilateral stimuli on each side correctly, but failed to perceive the left stimulus during bilateral stimulation in $>50 \%$ of the trials.

\section{RESULTS}

Ninety nine $(77 \%)$ of the 128 brain damaged subjects with left or right sided hemiparesis or hemiplegia mentioned their motor deficit spontaneously following a general question about their complaints. Twenty nine patients (23\%) were thus rated as showing mild, medium, or severe "anosognosia" for hemiparesis according to the anosognosia scale of Bisiach et al. ${ }^{3}$ Seventeen (59\%) of these 29 patients had a denial grade of 1 (that is, they reported their motor impairment only following a specific question about the strength of their limbs); eight $(27 \%)$ had a denial grade of 2 (recognising their paresis only after demonstration); and four ( $14 \%$ ) had a denial grade of 3 (remaining unaware of their deficit even after demonstration). Clinical and demographic variables of these patient groups are given in table 1 .

One might assume that the classification of a verbal behaviour scored as denial grade 1, representing mild anosognosia, ${ }^{36}$ could be an overinterpretation leading to an artificially high percentage of "anosognosia" in a sample of stroke patients. If such behaviour is instead regarded as nonpathological, one would expect these patients not to differ in their clinical variables from those with a denial grade of $0-$ that is, those with no anosognosia. Further, we would expect that such patients in denial grade 1 would differ from those in denial grades 2 or 3, as both latter groups show consistent failure to acknowledge hemiparesis or hemiplegia following a specific question about the strength of their limbs (subjects 
Table 2 Comparison of clinical variables between the patient groups with different denial grades (grades 0 to 3 according to the anosognosia scale of Bisiach et $a l^{3}$ )

\begin{tabular}{llll}
\hline & Denial grade & & \\
\cline { 2 - 4 } & Grade 0 v 1 & Grade 1 v 2/3 & Grade 0 v 2/3 \\
\hline Age & $p=0.555$ & $p=0.710$ & $p=0.300$ \\
Side of lesion & $p=0.272$ & $p=0.023$ & $p=0.065$ \\
Paresis of contralesional side & & & \\
$\quad$ Arm & $p=0.154$ & $p=0.030$ & $p<0.001^{*}$ \\
Leg & $p=0.062$ & $p=0.012 *$ & $p<0.001^{*}$ \\
Visual field defects & $p=0.597$ & $p=0.345$ & $p=0.669$ \\
Neglect & $p=0.470$ & $p=0.002^{*}$ & $p<0.001^{*}$ \\
Visual extinction & $p=0.063$ & $p=0.033$ & $p<0.001^{*}$ \\
Tactile extinction & $p=0.548$ & $p=0.049$ & $p<0.001^{*}$ \\
Auditory extinction & $p=0.969$ & $p=0.001^{*}$ & $p<0.001^{*}$ \\
Aphasia & $p=0.323$ & $p=0.070$ & $p=0.163$ \\
MMSE & $p=0.001^{*}$ & $p=0.258$ & $p<0.001^{*}$ \\
\hline *Significant with a Bonferroni corrected $\alpha$ level: $p=0.0167$. & & \\
MMSE, mini-mental state examination. & & & \\
\hline
\end{tabular}

in denial grade 2 only acknowledge hemiparesis/hemiplegia after demonstration; subjects in denial grade 3 do not acknowledge it at all). For the following analyses, we thus amalgamated patients in denial grades 2 and 3 .

In fact, comparison of the patients in denial grade 1 (the 17 patients who first reported their motor impairment after a specific question about the strength of their limbs) with those in denial grade 0 revealed a significant difference only for the MMSE score ( $U=410.5 ; p<0.001)$ (table 2$)$. All other variables such as age, severity of hemiparesis, aphasia, visual field defects, neglect, and extinction were comparable between the two groups. In contrast, comparison of patients in denial grade 1 with those in denial grades 2 or 3 showed more severe paresis of the lower limb $(U=48.0$; $\mathrm{p}<0.001)$, a higher incidence of spatial neglect $\left(\chi^{2}=9.64\right.$; $\mathrm{df}=1 ; \mathrm{p}=0.002)$, and more frequent auditory extinction $\left(\chi^{2}=10.71 ; \mathrm{df}=1 ; \mathrm{p}<0.001\right)$ in the latter groups. (These differences were still present if patients in grade 1 were compared only with those in grade 2.) Comparison of patients rated grade 0 with those in grade 2 or 3 revealed significant differences in the severity of upper and lower limb paresis, visual, tactile, and auditory extinction, spatial neglect, and the MMSE score. Table 2 summarises the statistical results of all these comparisons.

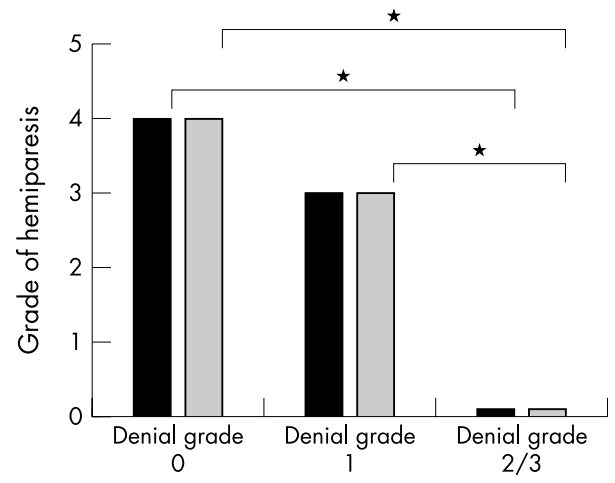

Figure 1 Median severity of contralesional hemiparesis. The degree of paresis was scored separately for the proximal and distal parts of each limb using the usual clinical ordinal scale, where 0 is no trace of movement and 5 is normal movement. Scores were averaged per limb. Black bars indicate scores for the upper limb; grey bars for the lower limb. Anosognosia for hemiplegia was examined using a German translation ${ }^{14}$ of the anosognosia scale suggested by Bisiach et al. ${ }^{3}$ Asterisks indicate significant differences using a Bonferroni corrected $\alpha$ level of $p=0.0167$.
We wondered whether the subjects in denial grade 1 - that is, who reported their motor impairment first after a specific question about the strength of their limbs-might not have mentioned their paretic limb spontaneously because the paresis was relatively mild and their other neurological symptoms were subjectively more prominent. Our investigations showed that these patients did indeed have significantly less severe paresis than those in denial grade 2 or 3 (fig l).

To evaluate whether the patients in denial grade 1 mentioned other neurological defects or symptoms that might have a greater personal impact for them, we analysed the verbal responses of all 17 subjects. The responses were classified into four categories and each patient was assigned to one of these categories (table 3). Following the general question about their complaints, the majority of the 17 patients mentioned other deficits like "I had difficulty in talking" or "my hand and leg felt numb" (table 3). One male patient with hypertrophy of his prostate mentioned that he had problems with his prostate and pain when he urinated. All these complaints were real. Four further patients complained about general symptoms like "tiredness", "headache", or "feeling sick" (table 3). Two patients had no memory for the stroke event, and one claimed that he came to the hospital "with the help of the ambulance after having a stroke". This latter response was categorised as "inappropriate" in table 3 as she mentioned the way in which she reached hospital but not the reason for being admitted. All

Table 3 Categories of responses given by the 17 patients in denial grade 1 following a general question about their complaints (the first level of severity of anosognosia in the anosognosia scale of Bisiach et al ${ }^{3}$ )

\begin{tabular}{|c|c|}
\hline Category & Response \\
\hline $\begin{array}{l}\text { Other deficits } \\
\text { Patients reported speech arrest, dysarthria, sensory } \\
\text { deficit, ptosis, facial paresis, etc }\end{array}$ & $59 \%(n=10)$ \\
\hline $\begin{array}{l}\text { General symptoms } \\
\text { Patients reported tiredness, dizziness, headache, etc }\end{array}$ & $23 \%(n=4)$ \\
\hline $\begin{array}{l}\text { No memory } \\
\text { Patients reported having no memory for the stroke ever }\end{array}$ & $12 \%(n=2)$ \\
\hline Inappropriate response & $6 \%(n=1)$ \\
\hline
\end{tabular}

The response of each of the 17 patients was assigned to only one of the four categories. 
the 17 patients recognised their paresis at once when the examiner asked them about the strength of their limbs.

\section{DISCUSSION}

In this study we investigated whether patients who do not mention their neurological defect (hemiparesis) spontaneously but only when asked about it directly by the examiner should be regarded as anosognosic. In particular, we asked whether hemiparetic stroke patients diagnosed as being in denial grade 1 according to the anosognosia scale of Bisiach et al should be classed as having "mild anosognosia" for hemiparesis or should be considered non-pathological.

Previous studies have shown that patients with anosognosia differ significantly from those without this feature with respect to the side of the lesion, additional neglect, and presence of extinction. ${ }^{467}$ In contrast, in the present study we found a high degree of similarity between patients in denial grade 0 (no anosognosia for hemiparesis) and those in denial grade 1. Except for the MMSE score, there were no significant differences between these two groups for any of the clinical variables tested.

The question arises as to why patients rated grade 1 did not mention their paresis spontaneously. Is it possible that they simply did not mention their paretic limb because the paresis was mild or because other deficits were subjectively more prominent? We found that the paresis of patients in denial grade 1 was indeed significantly milder than in denial grades 2 and 3. Moreover, analysis of the verbal responses showed that $59 \%$ of the patients in denial grade 1 complained spontaneously about other neurological deficits such as speech arrest, dysarthria, sensory deficit, ptosis, and so on. All these symptoms were real. An additional 35\% of the grade 1 group spontaneously mentioned symptoms such as tiredness, dizziness, headache, and loss of memory for the stroke event. Such symptoms are indeed common in stroke patients $^{16}{ }^{17}$ and thus may be assumed to be real and not just a pretense. Thus the data suggest that $94 \%$ of those patients rated as being in denial grade 1 did not appear to have a problem in accepting their hemiparesis but simply perceived other symptoms as being more prominent when asked a general question about their complaints. When the examiner asked about the strength of their limbs, all these patients immediately acknowledged their paresis. In contrast, when the patients in denial grade 2 and 3 were asked specifically about the strength of their limbs, they insisted that everything was all right. In conclusion, we think that a denial grade of at least 2 is the appropriate cut off point for a diagnosis of anosognosia for hemiparesis.

If we consider denial grade 1 to be non-pathological and only regard grades 2 and 3 as indicating anosognosia, our study gives an incidence of $10 \%$ for anosognosia for hemiparesis in a sample of 128 acute stroke patients. Applying the same cut off post hoc to previous studies on this issue leads to a more homogeneous incidence of anosognosia in patients with hemiparesis. Starkstein et $a l^{6}$ investigated a sample of 80 patients for anosognosia for hemiparesis. If these investigators had considered only those patients in denial grade 2 or 3 as anosognosic, the incidence of anosognosia for hemiparesis in that study would have been $18 \%$. Appelros et al, ${ }^{9}$ who used the same anosognosia scale as Starkstein et al, would similarly have obtained an incidence of $12 \%$.
In conclusion, we suggest that patients who do not mention their paresis spontaneously following a general question about their complaints but acknowledge it when asked about it directly should not be considered as having anosognosia. Our study showed that most patients in this category suffer from and report other deficits which are obviously more prominent for them. These patients have significantly milder paresis than those who deny their disorder even when they are asked specifically about their limbs; moreover, the clinical variables found in such patients do not differ from those in patients who report their disorder spontaneously.

If the present cut off criterion is applied post hoc to previous datasets from other investigators, the incidence of anosognosia for hemiparesis ranges from $10 \%$ to $18 \%$ in unselected samples of acute stroke patients with hemiparesis or hemiplegia. The incidence of anosognosia thus seems to be lower than previously assumed. ${ }^{3689}$

\section{ACKNOWLEDGEMENTS}

This work was supported by a grant of the Deutsche Forschungsgemeinschaft (Ka 1258/2-3).

\section{Authors' affiliations}

B Baier, H-O Karnath, Centre of Neurology, Hertie-Institute for Clinical Brain Research, University of Tübingen, Tübingen, Germany

Competing interests: none declared

\section{REFERENCES}

1 Bisiach E, Geminiani G. Anosognosia related to hemiplegia and hemianopia. In: Prigatano GB, Schacter DL, eds. Awareness of deficit after brain injury. New York: Oxford University Press, 1991:17-39

2 Heilman KM. Anosognosia: possible neuropsychological mechanisms. In: Prigatano GB, Schacter DL, eds. Awareness of deficit after brain injury. New York: Oxford University Press, 1991:53-62.

3 Bisiach E, Vallar G, Perani D, et al. Unawareness of disease following lesions of the right hemisphere: anosognosia for hemiplegia and anosognosia for hemianopia. Neuropsychologia 1986;24:471-82.

4 Cutting J. Study of anosognosia. J Neurol Neurosurg Psychiatry 1978;41:548-55

5 Maeshima S, Dohi N, Funahashi K, et al. Rehabilitation of patients with anosognosia for hemiplegia due to intracerebral hemorrhage. Brain Injury 1997:11:691-7.

6 Starkstein SE, Fedoroff JP, Price TR, et al. Anosognosia in patients with cerebrovascular lesions. Stroke 1992;23:1446-53.

7 Nathanson M, Bergman P, Gordon G. Denial of illness. Its occurrence in one hundred consecutive cases of hemiplegia. Arch Neurol Psychiatry 1952;68:380-7.

8 Stone SP, Halligan PW, Greenwood RJ. The incidence of neglect phenomena and related disorders in patients with an acute right or left hemisphere stroke. Age Aging 1993;22:46-52.

9 Appelros $\mathrm{P}$, Karlsson GM, Seiger A, et al. Neglect and anosognosia after firstever stroke: incidence and relationship to disability. J Rehabil Med 2002;34:215-20

10 Critchley M. The problem of awareness or non-awareness of hemianopic field defects. Trans Ophthalmol Soc UK 1949;69:95-109.

11 Feinberg TE, Roane DM, Kwan PC, et al. Anosognosia and visuoverbal confabulation. Arch Neurol 1994;51:468-73.

12 Jehkonen $M$, Ahonen J-P, Dastidar $P$, et al. Unawareness of deficit after right hemisphere stroke: double-dissociations of anosognosia. Acta Neurol Scand 2000;102:378-84.

13 Folstein MF, Folstein SE, McHugh PR. "Mini-mental state": a practical method for grading the cognitive state of patients for the clinician. J Psychiatr Res 1975; 12:189-98

14 Karnath H-O. Anosognosie. In: Hartje W, Poeck K, eds. Klinische Neuropsychologie, 5th ed. Stuttgart: Thieme Verlag, 2002:361-71.

15 Gauthier L, Dehaut $F$, Joanette $Y$. The bells test: a quantitative and qualitative test for visual neglect. Int J Clin Neuropsychol 1989;11:49-54

16 Rathore SS, Hinn AR, Cooper LS, et al. Characterization of incident stroke signs and symptoms. Stroke 2002;33:2718-21.

17 Handschu R, Poppe R, Rauß J, et al. Emergency calls in acute stroke. Stroke 2003;34:1005-9. 